\title{
3D Femur Reconstruction Using X-Ray Stereo Pairs
}

\author{
Sonia Akkoul ${ }^{1}$, Adel Hafiane ${ }^{2}$, Eric Lespessailles ${ }^{3}$, and Rachid Jennane ${ }^{1}$ \\ ${ }^{1}$ Univ. Orléans, PRISME, EA 4229, 12 rue de Blois BP 6744, F-45067 Orléans, France \\ ${ }^{2}$ ENSI de Bourges, Univ. Orléans, PRISME, EA 4229, \\ 88 avenue Lahitolle F-18020 Bourges, France \\ ${ }^{3}$ Univ. Orléans, I3MTO, EA 4708, CHR d'Orléans, \\ 1 rue Porte Madeleine F-45032 Orléans, France
}

\begin{abstract}
In this paper, we present a 3D reconstruction method for the shape of the proximal femur using pairs of $2 \mathrm{D}$ radiographs. The femur shape reconstruction from a small number of images is a challenging task but it is desired as it lowers both the acquisition costs and the radiation dose compared to tomography. In this paper we investigate the reconstruction of the 3D proximal femur surface without any prior acknowledge and using a limited number of $2 \mathrm{D}$ images. The proposed method uses a contour points coordinates and compares three different distances to find the best matching between 2D point pairs. The impact of varying the angles between the selected images on the reconstructed 3D shape is tested. Obtained results show that it is possible to rebuild the proximal femur shape from a limited number of radiographs.
\end{abstract}

Keywords: Stereo reconstruction, contour matching, proximal femur.

\section{Introduction}

Shape reconstruction from sparse set of 2D projection images is important for preoperative planning of several images guided surgical procedures. 3D reconstruction of anatomical models can be achieved using the direct 3D imaging modalities such as Computed Tomography (CT). However, the use of such imaging is restricted to a minor specific procedures; due to constraints placed by cost, availability and radiation risk. Thus, the diagnostics and planning of many interventions still rely on two dimensional (2D) radiographic images, where the surgeon has to mentally visualize the 3D anatomy of interest. In order to assist the clinicians on their medical tasks, a direct $3 \mathrm{D}$ imaging must be developed, as an alternative to current pure 2D radiographs [1]. However, constructing a 3D bone surface model from a limited number of 2D X-ray images is a challenging task, especially when constructing a patient specific surface model of a bone. Moreover, for surgical application, high accuracy reconstruction is required. The error of reconstruction has to be in the range of surgical usability.

Algorithms for 3D reconstruction can be categorized either as 2D or 3D similarity based. The 2D similarity group uses 2D images for 3D model reconstruction [2]. The $3 \mathrm{D}$ similarity group is based on a prior knowledge of the $3 \mathrm{D}$ anatomical structure and uses 2D images [1]. 
Most of the studies require prior knowledge of the 3D anatomy model to guide the reconstruction process and to compensate the lack of information in $2 \mathrm{D}$ imaging modalities [1]. This information can be provided by the integration of a generic geometrical surface of the considered bone structure [3], [4], or by Statistical Shape Models (SSM) [5-7].

The goal of this paper is to study the influence of the contours matching on the accuracy of the 3D femur surface reconstruction from limited number of X-ray images taken from different orientations. Using a standard X-ray projective model, two successive projections are used to compute the coordinates of a $3 \mathrm{D}$ contour. After extracting the contour of the proximal femur on the 2D X-ray images, comes the matching process. This stage is a very important step for the $3 \mathrm{D}$ reconstruction, because it impacts the accuracy of the computed 3D coordinates. There exists several works for contours matching [8-10]. In this work, we considered non rigid contour corresponding; the matching between the points of two contours is performed with three different distances, the City-block, the Chess board and the Euclidian distance. The estimated point pairs are then used to compute a set of 3D points. The different distances were evaluated in terms of the minimum error on the $3 \mathrm{D}$ reconstructed volume; the one with the minimum error was retained for the rest of the experiments. Since we are using different pairs of images to compute the 3D points, it is worthwhile to study the influence of the X-ray imaging angle between two pairs and between the images of each pair.

To assess the accuracy of the proposed shape reconstruction framework, the results were compared to real 3D CT scan data. The cadaver proximal femur was used as the anatomy of interest throughout this study.

This paper is organized as follows; next section describes the materials and methods. Section 3 presents the experiments and the different results. A discussion concludes this paper.

\section{Materials and Methods}

Using few X-ray image projections, our task is to establish correspondence between stereo pairs of input images. For this purpose, a cadaver proximal femur was completely scanned using a CT scanner. This modality of imaging provides both the projections and the resulting $3 \mathrm{D}$ volume. In addition, there is no need to calibrate the input images for the framework of our study. X-Ray tomographic images were obtained using a high resolution Computed Tomography (CT) scanner (VISCOM X8060 NDT). The cadaveric proximal femur was placed between the X-ray source and the sensor on a rotating stage to obtain projections at different angles. 450 radiographs (projections) with a size of $1024 \times 1024$ pixels were acquired on 360 degrees, which yield one projection at each 0.8 degree. The pixel size was $200 \mu \mathrm{m}$. Examples of the ex vivo proximal femur radiographs are shown in Fig. 1. The 3D volume was reconstructed with the complete set of the projections using Feldkamps cone beam reconstruction algorithm [11], the 3D model of the reconstructed proximal femur is shown in Fig. 2. This model has served as the ground truth to compare the performances of our proposed algorithm. 


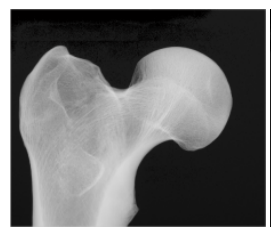

$0^{\circ}$

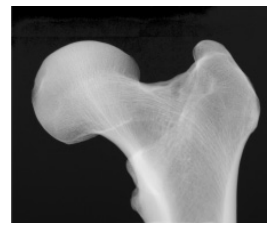

$220^{\circ}$

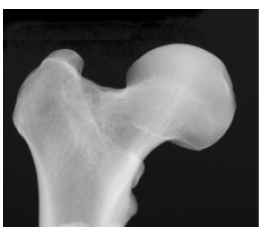

$44^{\circ}$

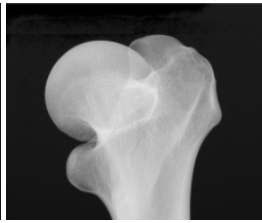

$264^{\circ}$

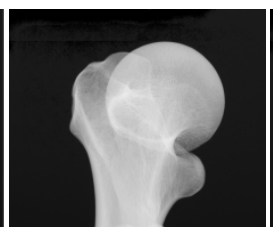

$92^{\circ}$

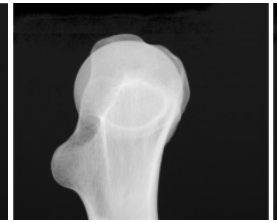

$292^{\circ}$

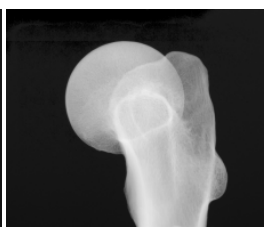

$136^{\circ}$

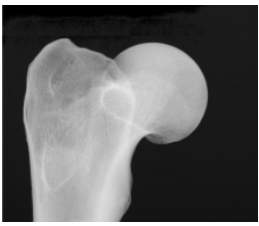

$336^{\circ}$

Fig. 1. X-ray images of the ex vivo proximal femur at different angles

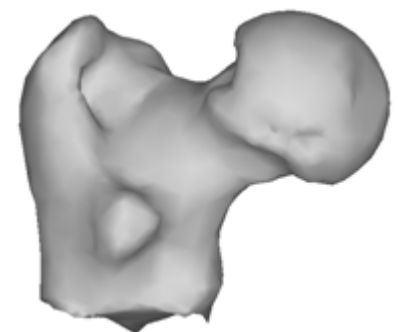

Fig. 2. 3D ground truth of proximal femur used for the test and reconstructed from 450 radiographs using X-ray tomography imaging

\subsection{Stereo from X-Ray}

The classical stereo techniques based on multiview matching points assume that the points in the object surface must be visible in all views. Theoretically, this assumption is not valid for the X-ray imaging modality, because the intensity values in the images are related to the X-ray penetration properties of the solid object and not to the reflection of the surface. However, under the general concept, each point of the contour in the projected images represents the intersection of the X-ray with surface at one point. This leads to preserve the surface visual properties, and the classical stereo assumption may apply under certain conditions. Nevertheless, there could be important surface occlusion, when the angle between two radiographs is large. In this work we assume that the occlusion is neglected if the angle between two projections is small. Therefore, we are interested in the impact of the angle between two radiographs as well as the influence of the chosen step between two pairs of radiographs on the quality and the accuracy of the 3D reconstructed shape. To do so, the angle, $\alpha$, between two images of the same pair was fixed to 4 degrees and the angle, $\beta$, between two pairs was set to different values $10,20,30,45$ and 60 degrees. For each angle $\beta$, the 
error between the 3D reconstructed shape and the ground truth was estimated and the optimal orientation $\beta_{o}$ giving the minimum error was retained. Keeping the angle $\beta_{o}$, the next step consisted in varying the angle $\alpha$ from 4 to 32 degrees by power of 2 , in order to get the best combination $\left(\alpha_{o}, \beta_{o}\right)$ that enables reconstructing the $3 \mathrm{D}$ desired shape close to the ground truth. Fig. 3 illustrates different pairs of X-ray projections separated by the angles $\alpha$ and $\beta$.

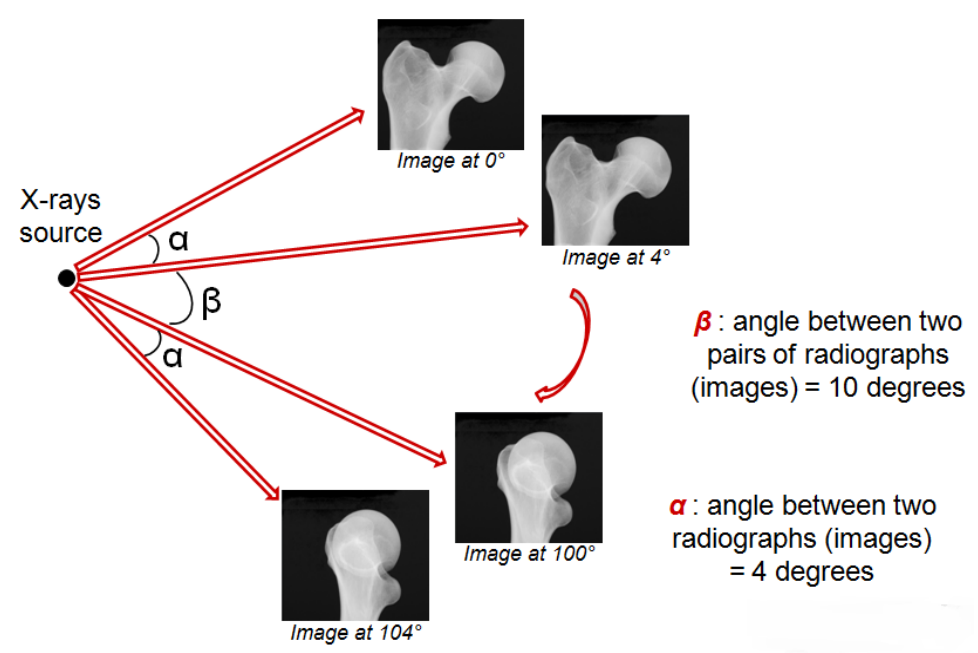

Fig. 3. Illustration of the angle $\beta$ between two pairs of radiographs as well as the angle $\alpha$ between two radiographs of the same pair

The next sections describe the processing methods that are used before computing the $3 \mathrm{D}$ coordinates of the target $3 \mathrm{D}$ shape.

\subsection{Contour Points Matching}

Here the objective is to establish correspondence between the two input images. For a given point on the first X-ray image, the goal is to identify a corresponding point on the second X-ray image. As discussed previously, we are interested only by the proximal femur surface, in results the matching is realized using only the contours of the shape. Edge localization determines the precision of the 3D coordinates estimation. In our case, the X-ray imaging makes the border fuzzy difficult to define the real position of the edges. We have applied a morphology closing operation to the binarized $\mathrm{X}$-ray images. The binarization consisted in determining a threshold based on the local minimum between the two modes of the histogram of each image [12].

Once the contours were detected, points from the two contours have to be matched and associated. Both associated points have to be as close as possible to each other. For this purpose, we have tested three different distances, the City-block, the Chess-board and the Euclidean Distance. 
Let us denote the coordinates of the detected edge pixels in image1 (red edge, Fig. 4) as $I_{1}=\left\{\left(x_{i}^{1}, y_{i}^{1}\right), i=0,1, \ldots, M-1\right\}$ and the detected edge pixels in image2 (green edge, Fig. 4) a $I_{2}=\left\{\left(x_{i}^{2}, y_{i}^{2}\right), i=0,1, \ldots, M-1\right\}$, where $\mathrm{M}$ and $\mathrm{N}$ are the number of the edge points detected in image 1 and in image 2 , respectively.

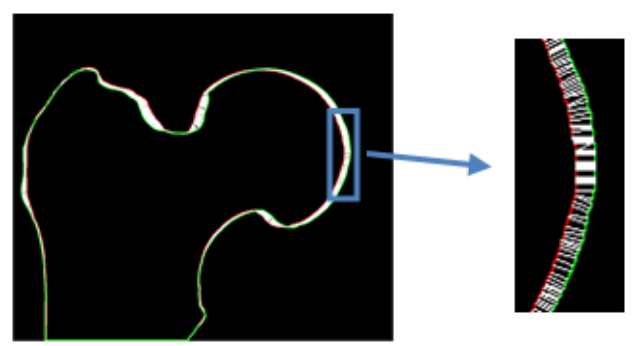

Fig. 4. Matched points using the Euclidean distance and the coordinates of the points

The matching process consists in finding for each point in the set $I_{1}$, the closest one in $I_{2}$, by computing the City-block $d_{l}$ as (1), the Chess-board $d_{2}$ as (2) and the Euclidean distance $d_{3}$ as (3).

$$
\begin{gathered}
d_{1}(x, y)=\left|x_{i}^{1}-x_{j}^{2}\right|+\left|y_{i}^{1}-y_{j}^{2}\right| \\
d_{2}(x, y)=\max \left(\left|x_{i}^{1}-x_{j}^{2}\right|\left|y_{i}^{1}-y_{j}^{2}\right|\right) \\
d_{3}(x, y)=\sqrt{\left(x_{i}^{1}-x_{j}^{2}\right)^{2}+\left(y_{i}^{1}-y_{j}^{2}\right)^{2}}
\end{gathered}
$$

The next section presents how to register the reconstructed form and the ground truth in the same environment for error estimation.

\subsection{D Points Registration and Meshing}

Before the meshing step of the obtained 3D points cloud, we applied a rigid registration to those points with a 3D points cloud extracted from the ground truth. We used the Iterative Closest Point (ICP) algorithm [12], which is employed to minimize the difference between two clouds of points. The first step of the ICP algorithm is based on the search of pairs of nearest points between the two sets (reconstructed and ground truth). The second step includes the estimation of the optimal rigid transformation that aligns the two data sets. Then, in the last step, the rigid transformation is applied to the points of the reconstructed data. The procedure iteratively revises the transformations (translation, rotation) needed to minimize the distance between the points until convergence is achieved. Registering the 3D points cloud in the same environment enables estimating the error between the two surfaces. Fig. 5 illustrates the obtained registration for the two data sets. 


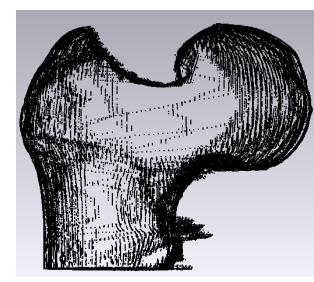

The computed 3D points cloud

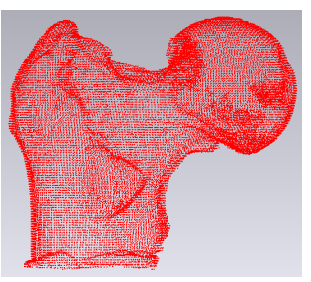

The ground truth points cloud
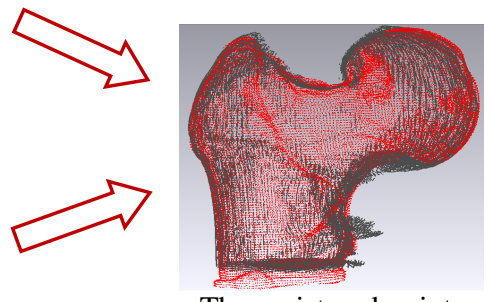

The registered points

cloud (ICP)

Fig. 5. Registration of the 3D point clouds from the ground truth and the reconstructed surface

Next, comes the meshing and the reconstruction of the computed 3D points. This task was achieved using Meshlab [14] which is an advanced 3D software system for the processing and editing of unstructured 3D triangular meshes. Based on the normal's of the points, we used the Poisson surface reconstruction algorithm proposed by Meshlab. Fig. 6 presents the result.

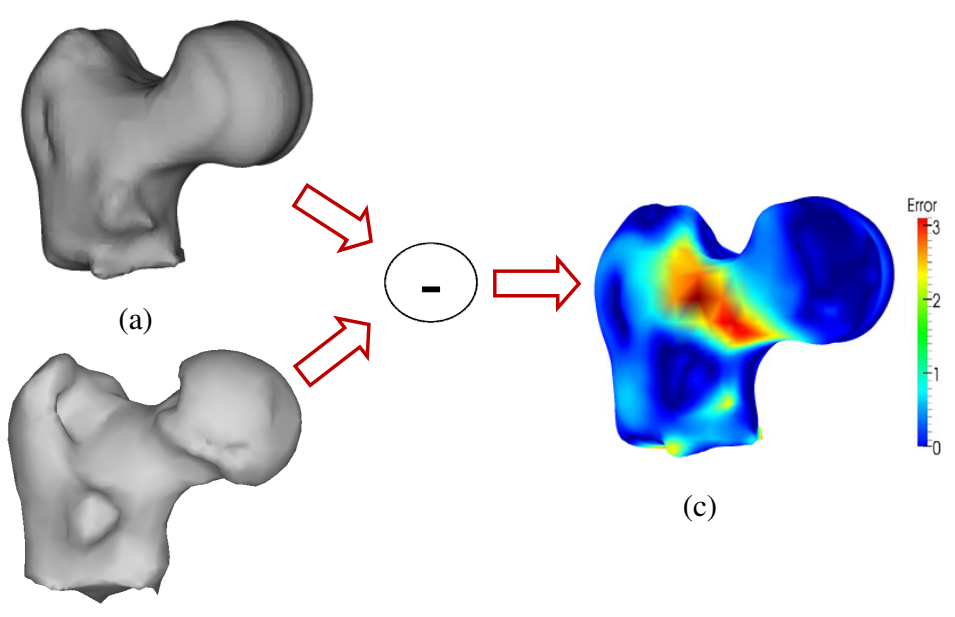

(b)

Fig. 6. (a) The computed 3D shape surfaces (Meshlab), (b) the ground truth surfaces (Meshlab), (c) 3D surfaces differences and error estimation (Metro tool) 


\section{$3 \quad$ Results}

First, we compared the performances with three distances, $\beta$ and $\alpha$ were settled to 10 and 4 degrees respectively. The $\beta$ parameter determines the number of tridimensional contours that we can obtain from the image pairs; for instance, $\beta=10$ degrees yields 36 contours in 3D space. Taking radiographs pairs from all directions (360 degrees) ensures including maximum information reflecting the different structures of the proximal femur. Fig. 7 presents the results of our 3D reconstruction technique using the three distances. The estimated reconstruction errors obtained in comparison to the ground truth are presented too. These errors were evaluated using the Metro tool for measuring error between simplified surfaces [15]. The red and blue colors in the 3D reconstructed shapes represent respectively the high and the low error values. Errors are minimal in the third case where the euclidean distance is used.

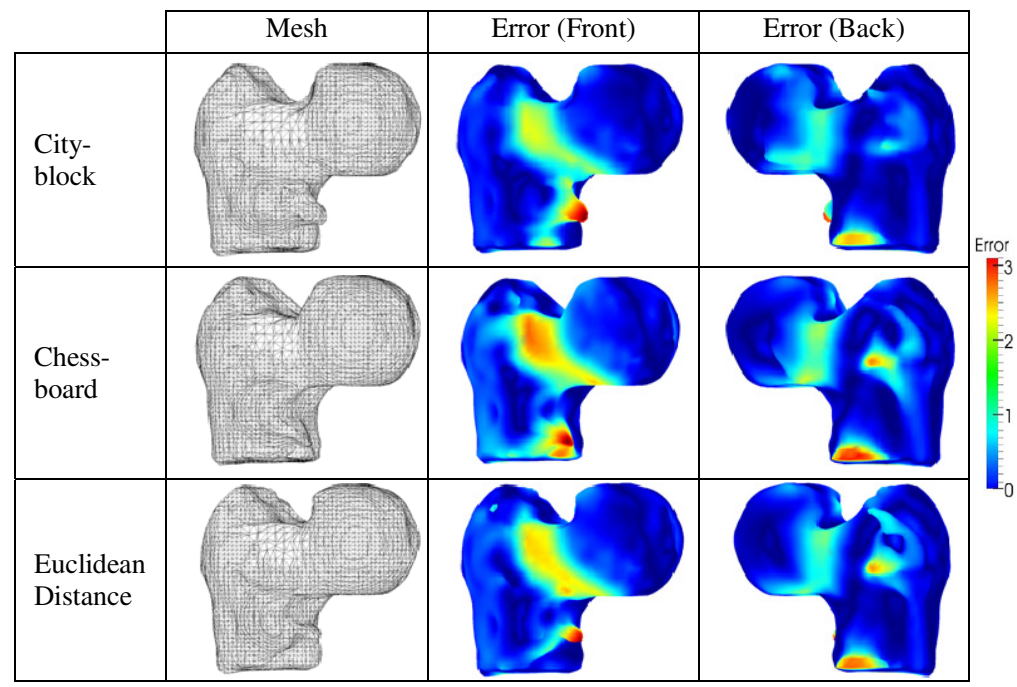

Fig. 7. The first column presents 3D meshed shapes (Meshlab) of the computed points using the three distances; the second and third columns show the corresponding errors (front and back views) to the ground truth (using Metro tool)

For quantitative evaluation of the performances of the different distances, we computed the mean error and the Root Mean Square (RMS) error for the three distances. Fig. 8 shows the results of both estimated errors for the different distances. The Euclidean distance yields the best results, thus it is retained for the next experiments that test the effect of the angle $\beta$ on the reconstructed 3D surface, the angle $\alpha$ was set to $4^{\circ}$. We considered the following orientations: $10,20,30,45$ and 60 degrees which correspond to $36,18,12,8$ and 6 pairs of projections respectively. The obtained mean and RMS errors for the different orientations $\beta$ are given in Fig. 9. Surprisingly, $\beta=45^{\circ}$ yields the minimum mean and RMS errors. 


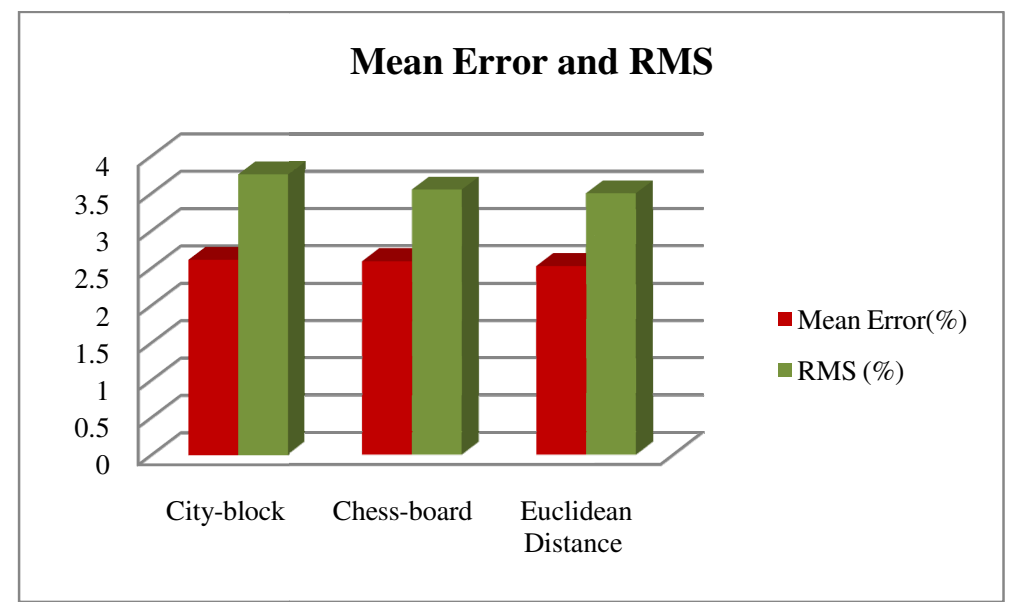

Fig. 8. The mean error and the RMS error for the three distances

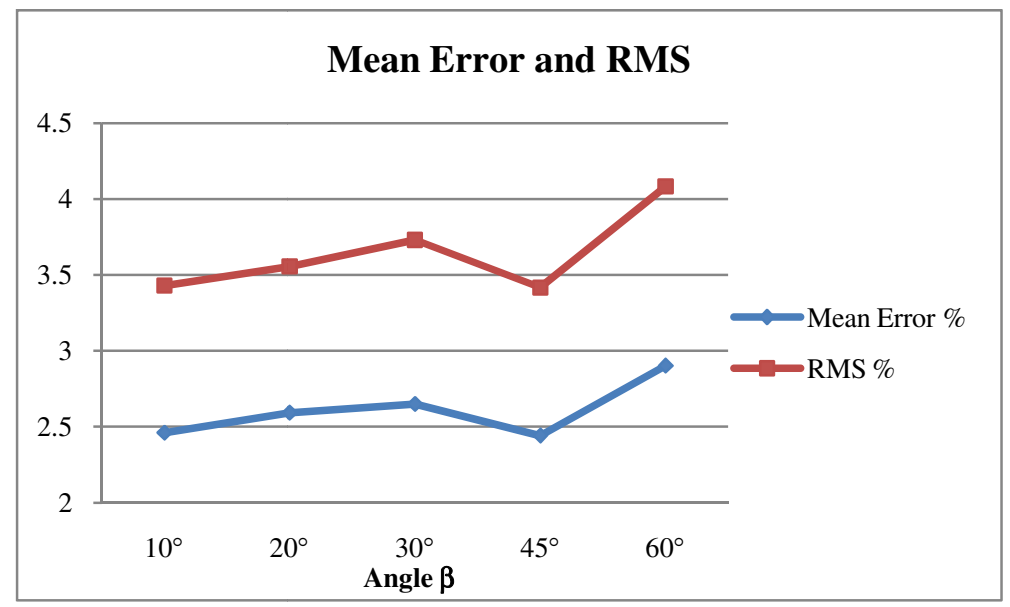

Fig. 9. The quantitative reconstruction error for the different angles between radiographs pairs

Once the optimal orientation $\beta_{\mathrm{o}}$ was obtained, we looked for the optimal value $\alpha_{\mathrm{o}}$. This was realized by fixing $\beta$ to $45^{\circ}$ and varying the angle $\alpha$ from 4 to 32 degrees by power of 2. The obtained results are shown in Fig. 10.

The angle of 4 degrees gives the minimum mean error and RMS error. These results are consistent with our expectations, closer are the projections for estimation of the $3 \mathrm{D}$ contour points, best is the contour matching and minimum is the error. As a conclusion, the optimal angles for the 3D reconstruction of the proximal femur shape is $\left(\alpha_{\mathrm{o}}, \beta_{\mathrm{o}}\right)=\left(4^{\circ}, 45^{\circ}\right)$ which lead to the use of 16 projections. 


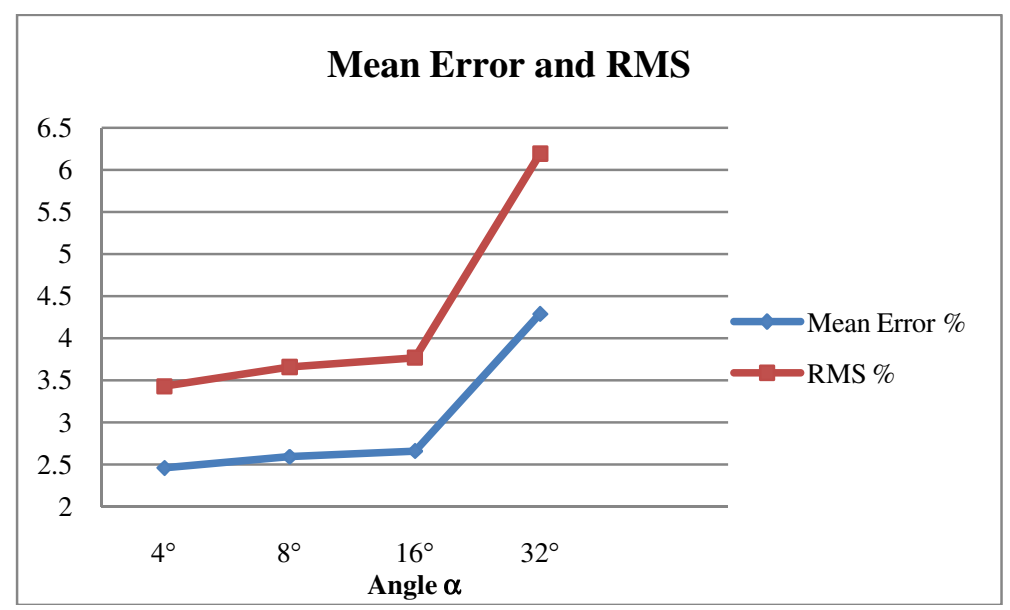

Fig. 10. The mean Error and the RMS error for the different angles

\section{Discussion and Conclusion}

In this paper, we presented a simple method for $3 \mathrm{D}$ reconstruction of the femur shape from relatively small number of X-ray projections. The proposed reconstruction scheme combines X-ray stereo model and contour points matching. For the point matching, the impact of three different distances was studied, the City-block, the Chess-board and the Euclidean Distance. The obtained results showed that the Euclidian Distance is well suited for our experiment. Compared to other previous methods, our technique differs in the way how the contours are combined, assuming that the contours in the X-ray projection follow the traditional stereo condition under small changes in the projection angle. Images were selected by pairs for contour matching. The effects of the angles between the pairs and between the images of the same pair were tested. As expected, results showed that the angle between pairs of images has to be well identified to capture the main forms of the femur. While the angle between the images of the same pair has to be minimal for a good estimation of the $3 \mathrm{D}$ coordinates of the contour.

To assess the feasibility of our preliminary study, we used a cadaver proximal femur and its CT scan data. Further experiments using 2D classical X-ray images should be conducted to evaluate the effective potential of our proposed method.

Intra-operative assistance plays important roles in planning and supporting various computer assisted surgical procedures, we believe that our proposed approach shows promise for clinical applications.

Acknowledgement. The authors gratefully acknowledge the financial support provided by the Région Centre, France, under the FRACTOS project. 


\section{References}

1. Gamage, P., Xie, S.Q., Delmas, P., Xu, W.L.: Diagnostic radiograph based 3D bone reconstruction framework: Application to the femur. Comput. Med. Imaging Graph. 35(6), 427-437 (2011)

2. Caponetti, L., Fanelli, A.M.: Computed-aided simulation for bone surgery. IEEE Comput. Graph. Appl. 13, 86-92 (1993)

3. Laporte, S., Skalli, W., De Guise, J.A., Lavaste, F., Mitton, D.: A biplanar reconstruction method based on 2D and 3D contours: application to the distal femur. Comput. Methods Biomech. Engin. 6(1), 1-6 (2003)

4. Le Bras, A., Laporte, S., Bousson, V., Mitton, D., De Guise, J.A., Laredo, J.D., Skalli, W.: $3 \mathrm{D}$ reconstruction of the proximal femur with low-dose digital stereoradiography. Comput. Aided Surg. 9(3), 51-57 (2004)

5. Baka, N., Kaptein, B.L., de Bruijne, M., van Walsum, T., Giphart, J.E., Niessen, W.J., Lelieveldt, B.P.F.: 2D-3D shape reconstruction of the distal femur from stereo X-ray imaging using statistical shape models. Medical Image Analysis 15(6), 840-850 (2011)

6. Whitmarsh, T., Humbert, L., De Craene, M., Del Rio Barquero, L.M., Frangi, A.F.: Reconstructing the 3D Shape and Bone Mineral Density Distribution of the Proximal Femur From Dual-Energy X-Ray Absorptiometry. IEEE Transactions on Medical Imaging 30(12) (2011)

7. Zheng, G., Gollmer, S., Schumann, S., Dong, X., Feilkas, T.: A 2D/3D correspondence building method for reconstruction of a patient-specific 3D bone surface model using point distribution models and calibrated X-ray images. Medical Image Analysis 13(6), 883-899 (2009)

8. Cui, M., Femiani, J., Hu, J., Wonka, P., Razdan, A.: Curve matching for open 2D curves. Journal Pattern Recognition Letters 30(1), 1-10 (2009)

9. Frenkel, M., Basri, R.: Curve matching using the fast marching method. In: Rangarajan, A., Figueiredo, M.A.T., Zerubia, J. (eds.) EMMCVPR 2003. LNCS, vol. 2683, pp. 35-51. Springer, Heidelberg (2003)

10. Park, J.S., Han, J.H.: Contour matching: a curvature-based approach. Image and Vision Computing 16(3), 181-189 (1998)

11. Feldkamp, L.A., Davis, L.C., Kress, J.W.: Practical cone-beam algorithm. J. Opt. Soc. Am. A 1(6), 612-619 (1984)

12. Chappard, C., Marchadier, A., Benhamou, C.L.: Side-to-side and within-side variability of $3 \mathrm{~d}$ bone microarchitecture by conventional micro-computed tomography of paired iliac crest biopsies. Bone 43(1), 203-208 (2008)

13. Andette, M., Ferrie, F., Peters, T.: An Algorithmic Overview of Surface Registration Techniques for Medical Imaging. Medical Image Analysis 4, 201-217 (2000)

14. Meshlab, http://meshlab. sourceforge.net

15. Cignoni, P., Rocchini, C., Scopigno, R.: Metro: measuring error on simplified surfaces. Computer Graphics Forum, Blackwell Publishers 17(2), 167-174 (1998),

http://vcg.sf.net 